\title{
The higher dimensional Myers-Perry black hole with single rotation always obeys the cosmic censorship conjecture
}

\author{
Sanjar Shaymatov ${ }^{1,2, a}$, Naresh Dadhich ${ }^{3, b}$, Bobomurat Ahmedov ${ }^{1,4, c}$ \\ ${ }^{1}$ Ulugh Beg Astronomical Institute, Astronomicheskaya 33, Tashkent 100052, Uzbekistan \\ ${ }^{2}$ Institute of Nuclear Physics, Ulughbek, Tashkent 100214, Uzbekistan \\ ${ }^{3}$ Inter University Centre for Astronomy and Astrophysics, Post Bag 4, Pune 411007, India \\ ${ }^{4}$ National University of Uzbekistan, Tashkent 100174, Uzbekistan
}

Received: 1 April 2019 / Accepted: 30 June 2019 / Published online: 11 July 2019

(C) The Author(s) 2019

\begin{abstract}
Even though the Myers-Perry five dimensional rotating black hole with two rotations could be overspun by test particle accretion, yet it turns out as we show in this letter that it cannot do so for a single rotation. On the other hand it is known that there exists no extremal limit for a black hole with single rotation in dimensions greater than equal to six. It has been proven that all higher dimensional ( $>4)$ rotating black holes with only one single rotation can never be overspun under test particle linear accretion and hence would always obey CCC in the weak form.
\end{abstract}

\section{Introduction}

The occurrence of singularity is a generic property of Einstein's gravity - general relativity (GR), which has been established by very powerful singularity theorems [1]. A singularity marks the limit of a theory's applicability where it loses its predictive power. Fortunately, singularity that results under gravitational collapse of an object that has exhausted all its resources to counter gravity, is covered by an event horizon of black hole. Hence it turns benign for the region lying outside black hole horizon because no signal could come out of horizon.

For validity and applicability of the Einstein gravity in the large, Penrose pronounced in 1969 [2] that this would always be the case in general - a singularity would always be hidden behind a horizon. This is what is called the cosmic censorship conjecture (CCC) (all through by CCC we would mean weak CCC). There exists no proof of the conjecture either way, true or false, and it remains as one of

\footnotetext{
a e-mail: sanjar@astrin.uz

be-mail: nkd@iucaa.in

c e-mail: ahmedov@astrin.uz
}

the most important open questions. It has two aspects, one whether gravitational collapse can never end in a naked singularity without a horizon cover, and two could an existing horizon of a charged/rotating black hole be destroyed by overcharging/rotating? Even though there exists a vast literature on the former [3-8], yet there is no consensus on the occurrence of naked singularity. However for generic conditions collapse of a differentially rotating neutron star suggests that $\mathrm{CCC}$ is not violated [9]. We shall however focus here on the latter aspect of destroying horizon of a near extremal black hole by impinging it with test particles of suitable parameter in a Gedanken experiment.

First the question addressed was, whether a non-extremal black hole could be turned extremal by particle accretion leading to black hole temperature reducing to zero - violation of the third law of black hole thermodynamics? It was shown $[10,11]$ that it was not possible because as extremality was approached the particle parameter space pinched off - particles with suitable parameters won't be able to reach horizon near extremality. Then the question was formulated somewhat differently, true, extremality may not be achievable, could it however be jumped over? That is, in a discontinuous manner a black hole could jump from sub-extremal to over-extremal state, without passing through extremality - rather jumping over it, thereby destroy horizon and create a naked singularity.

A near extremal charged black hole $Q<M$ was shown [12] to be overcharged to $Q>M$ state by accretion of overcharged test particles. It was a linear order process in which effects of self force as well as of finite size of particle were ignored. Following that the same result was extended to rotating black hole $[13,14]$ - a rotating black hole could similarly be overspun. Following $[12,13]$ there have been 
several investigations on these lines [15-20] addressing the question of over-charging/spinning of black hole. ${ }^{1}$

It turns out that horizon of a charged and or rotating black hole can be destroyed by particle accretion of suitable parameters, and a naked singularity can be created. In all these works, it was assumed that test particle follows a geodesic (Lorentz force trajectory for charged particle) motion, and contributions of backreaction, self force and radiative effects were not taken into consideration. If these effects are taken into account, particles that could overspin the black hole would not be able to fall into the horizon [22-27]. And so the CCC may indeed be respected.

All this was in the linear regime, and the question was open whether the result would stand when non-linear accretion regime was considered. That has recently been done [28] and the answer has come out in favor of $\mathrm{CCC}$, that black hole cannot be over-charged/spun - horizon cannot be destroyed. It has also been shown that the same is the case for Kerr-AdS black hole [29].

Another question then arises, what happens in higher dimensions - could a five dimensional black hole be overcharged or overspun? It is shown [30] that a five dimensional charged black hole could be overcharged for linear test particle accretion and the horizon is destroyed. Following [28], there have been some non-linear accretion studies [31] verifying the validity of CCC. The general picture that emerges is that for linear order accretion a black hole could be overcharged or overspun and CCC is violated while it could not be done so for non-linear accretion and thereby restoring CCC.

A rotating black hole in higher dimensions has more than one rotation axis; i.e. a five dimensional black hole could have two rotation parameters about the two axes. Linear as well as non-linear accretion process have been studied [32] for the five dimensional Myers-Perry rotating black hole [33], and it is shown that though CCC is violated for the linear accretion but it is as expected obeyed for the non-linear accretion. This was all done for black hole having two rotation parameters. Could the situation be different if black hole has only one single rotation? The answer to this question is surprisingly yes for a five dimensional rotating black hole under linear accretion. To investigate this question defines the purpose of this letter.

In this letter we wish to point out that the situation is radically different for a five dimensional black hole having a single rotation than the one having two rotations under linear accretion. It remarkably turns out that a black hole with single rotation could not be overspun for linear test particle

\footnotetext{
${ }^{1}$ In Ref. [15], it is essentially shown for different five dimensional black hole geometries that geodesic particle accretion can never lead to extremality. This is because parameter space required for attaining extremality pinches off as it is approached [10,11]. Very recently this calculation is also done for multi black hole system [21] showing the same result.
}

accretion, and thereby CCC is respected. This is in stark contrast with the four dimensional Kerr as well as the five dimensional Myers-Perry black hole with two rotations [32] where it could be overspun and CCC is violated for linear accretion. Besides our independent calculations, we have also verified the result by switching off one of rotation parameters in the Ref. [32] analysis which considers both linear and non-linear accretion for two rotations. The main result that emerges is that a five dimensional black hole with only one rotation cannot be overspun even at linear accretion. As expected, this result continues to be true for non-linear accretion. This is different from all other cases where at linear order overspinning is always possible. That is, CCC is violated at linear order which is restored only when non-linear accretion is invoked.

With this background the result stands out that five dimensional black hole with single rotation does not require nonlinear accretion for respecting CCC. Hence CCC is always respected for single rotation five dimensional black hole irrespective of accretion being linear or non-linear.

This happens because the minimum threshold angular momentum required for overspinning turns out to be greater than maximum threshold allowed for particle reaching horizon. Thus there is no parameter space available for test particles that could lead to overspinning of black hole. This is exactly what happens for extremal black hole, minimum threshold leading to over-extremality turns out to be greater than the corresponding maximum threshold. That is why an extremal horizon can never be destroyed - an extremal black hole can never be pushed to over-extremal state of naked singularity.

The paper is organized as follows: In Sect. 2, we briefly recall the five dimensional rotating black hole metric which is followed by discussion of over extremality of black hole and we show that five dimensional rotating black hole with a single rotation cannot be overspun even in linear accretion process in the Sect. 3. We end up with conclusion in the Sect. 4.

\section{Five dimensional Myers-Perry rotating black hole}

Further it is known [33] that a rotating black hole with a single rotation has no extremal limit in dimension $\geq 6$, and hence it cannot be overspun simply because it can have arbitrary angular momentum without destroying horizon. Combining this fact with the above result we arrive at a very important and interesting conclusion that a rotating black hole with single rotation in dimension greater than four always obeys CCC. We begin as follows:

Let's recall the metric of the five dimensional MyersPerry rotating black hole without cosmological parameter $\Lambda$ [34], 


$$
\begin{aligned}
d s^{2}= & -\frac{\Delta}{\Sigma}\left(d t-a \sin ^{2} \theta d \phi-b \cos ^{2} \theta d \psi\right)^{2}+\frac{\Sigma}{\Delta} d r^{2} \\
& +\Sigma d \theta^{2}+\frac{\sin ^{2} \theta}{\Sigma}\left[\left(r^{2}+a^{2}\right) d \phi-a d t\right]^{2} \\
& +\frac{\cos ^{2} \theta}{\Sigma}\left[\left(r^{2}+b^{2}\right) d \psi-b d t\right]^{2} \\
& +r^{2}\left(\cos ^{2} \theta+\sin ^{2} \phi\right) d \psi^{2},
\end{aligned}
$$

where $\Delta=\frac{\left(r^{2}+a^{2}\right)\left(r^{2}+b^{2}\right)}{r^{2}}-\mu$ and $\Sigma=r^{2}+a^{2} \cos ^{2} \theta+$ $b^{2} \sin ^{2} \theta$. Here $a=\frac{4 J_{\phi}}{\pi \mu}$ and $b=\frac{4 J_{\psi}}{\pi \mu}$ are rotation parameters about the two rotation axes, and $\mu=\frac{8 M}{3 \pi}$ is mass parameter.

The black hole horizon is given by

$$
\begin{aligned}
r_{ \pm}= & \left(\frac{1}{2}\right)^{1 / 2}\left[\left(\mu-a^{2}-b^{2}\right)\right. \\
& \left. \pm \sqrt{\left(\mu-a^{2}-b^{2}\right)^{2}-4 a^{2} b^{2}}\right]^{1 / 2},
\end{aligned}
$$

and extremality is indicated by $a+b=(\mu)^{1 / 2}$.

The necessary and sufficient conditions for overextremality(spinning) are: (a) An overspinning particle must fall into black hole; i.e. it reaches horizon. This will define the maximum threshold for particle angular momentum $\delta J_{\max }$ as given in Eqs. 3 and 4. This is the maximum angular momentum a particle can carry while falling into black hole. (b) On accretion resulting state of black hole should be overextremal/spun, that would define the minimum threshold, $\delta J_{\min }$ as given in Eqs. 5 and 6 . The parameter window $\Delta J=\delta J_{\max }-\delta J_{\min }$ defines the parameter space of accreting particle required for overspinning. A black hole can overspin if and only if $\Delta J>0$, and if the opposite, $\Delta J<0$, is true, it cannot be. In that case overspinning particle would not be able to reach horizon to fall into black hole. This is precisely what we shall show in the following that $\Delta J<0$ for a black hole having only one rotation parameter, and hence black hole cannot be overspun.

\section{Overspinning of five dimensional rotating black hole with test particles}

Let a particle of energy $\delta E$ and angular momenta $\delta J=\delta J_{\phi}+$ $\delta J_{\psi}$, fall into a black hole. For particle to reach horizon, we have $\delta E \geq \Omega_{+}^{(\phi)} \delta J_{\phi}+\Omega_{+}^{(\psi)} \delta J_{\psi}$, and so we write

$\delta E \geq \frac{a}{r_{+}^{2}+a^{2}} \delta J_{\phi}+\frac{b}{r_{+}^{2}+b^{2}} \delta J_{\psi}$,

where $\Omega_{+}^{(\phi)}$ and $\Omega_{+}^{(\psi)}$ are respectively two black hole angular velocities relative to $\phi$ and $\psi$ axes.
By writing $\delta J_{\phi}=\lambda \delta J, \delta J_{\psi}=\alpha \delta J$ with $\alpha+\lambda=1$ and the maximum threshold is defined by

$\delta J_{\text {max }}=\frac{\mu r_{+}^{2}}{a\left(r_{+}^{2}+b^{2}\right)(1-\alpha)+b\left(r_{+}^{2}+a^{2}\right) \alpha} \delta E$.

On the other hand minimum threshold would be given by

$\sqrt{\frac{8}{3 \pi}}(M+\delta E)^{1 / 2}<\frac{3}{2}\left(\frac{J_{\phi}+\delta J_{\phi}}{M+\delta E}+\frac{J_{\psi}+\delta J_{\psi}}{M+\delta E}\right)$,

and hence

$$
\begin{aligned}
\delta J_{\text {min }}= & \delta J_{\phi}+\delta J_{\psi}=\left(\sqrt{\frac{32}{27 \pi}} M^{3 / 2}-J_{\phi}-J_{\psi}\right) \\
& +\sqrt{\frac{32}{27 \pi}}\left(\frac{3}{2} M^{1 / 2} \delta E+\frac{3}{8} M^{-1 / 2} \delta E^{2}\right) .
\end{aligned}
$$

Note that an exactly extremal black hole can never be overextremalized simply because in that case $\delta J_{\max }<\delta J_{\min }$ leaving no parameter window available for over-extremality. This is why one has always to begin with a near extremal state, $J_{\phi}+J_{\psi}=\frac{2}{3}(a+b) M=\sqrt{\frac{32}{27 \pi}} M^{3 / 2}\left(1-\epsilon^{2}\right), \epsilon \ll 1$. We write $a=\sqrt{\frac{8 M}{3 \pi}} \gamma\left(1-\epsilon^{2}\right)$ and $b=\sqrt{\frac{8 M}{3 \pi}} \beta\left(1-\epsilon^{2}\right)$ with $\gamma+\beta=1$, then $\delta J_{\max }$ and $\delta J_{\min }$ are written as

$$
\begin{aligned}
\delta J_{\text {max }}= & {\left[1+\sqrt{2 \frac{(\alpha-\beta)^{2}}{(1-\beta) \beta}} \epsilon\right.} \\
& \left.+\frac{\left[2 \alpha^{2}(1-2 \beta)^{2}-\alpha\left(1-6 \beta+8 \beta^{2}\right)+\beta^{2}\right]}{(1-\beta) \beta} \epsilon^{2}\right] \\
& \times \sqrt{\frac{8}{3 \pi}} M^{1 / 2} \delta E, \\
\delta J_{\text {min }}= & \delta J_{\phi}+\delta J_{\psi} \\
= & \sqrt{\frac{8}{3 \pi}}\left(\frac{2}{3} M^{3 / 2} \epsilon^{2}+M^{1 / 2} \delta E+\frac{1}{4} M^{-1 / 2} \delta E^{2}\right),
\end{aligned}
$$

and

$$
\begin{aligned}
\Delta J= & \sqrt{\frac{8}{3 \pi}}\left(\left[\sqrt{2 \frac{(\alpha-\beta)^{2}}{(1-\beta) \beta}} \epsilon\right.\right. \\
& \left.+\frac{\left[2 \alpha^{2}(1-2 \beta)^{2}-\alpha\left(1-6 \beta+8 \beta^{2}\right)+\beta^{2}\right]}{(1-\beta) \beta} \epsilon^{2}\right] \\
& \left.\times M^{1 / 2} \delta E-\frac{2}{3} M^{3 / 2} \epsilon^{2}-\frac{1}{4} M^{-1 / 2} \delta E^{2}\right)
\end{aligned}
$$


For overspinning, $\Delta J>0$ which would define parameter window for $\delta E$ as given by

$$
\begin{gathered}
2\left(\sqrt{2 \frac{(\alpha-\beta)^{2}}{(1-\beta) \beta}}-\sqrt{2 \frac{(\alpha-\beta)^{2}}{(1-\beta) \beta}-\frac{2}{3}}\right)<\Delta E \\
\quad<2\left(\sqrt{2 \frac{(\alpha-\beta)^{2}}{(1-\beta) \beta}}+\sqrt{2 \frac{(\alpha-\beta)^{2}}{(1-\beta) \beta}-\frac{2}{3}}\right),
\end{gathered}
$$

where $\Delta E=\delta E / M \epsilon$. This shows that $\Delta J>0$ and there exists a parameter window available as shown above for overspinning the black hole. A black hole with two rotations could indeed be overspun. Thus the horizon would be destroyed leading to naked singularity, and CCC would be violated. This was what shown in Ref. [32].

Let's first consider the case of infalling particle having only one angular momentum, $J_{\psi}$; i.e $\alpha=1$, and black hole having the two rotations, then the above inequality would require $\beta<3 / 4$. Again there would be parameter window available for test particles for overspinning the black hole. That is, a black hole with two rotations could always be overspun so long as $\beta<3 / 4$. Clearly there would be no overspinning possible for $\beta>3 / 4$. Note that single rotation parameter means $\beta=1$ indicating that black hole cannot be overspun in that case. However, we cannot take this limit in the above inequality because the terms diverge, and hence we have to consider the case of single rotation afresh separately. That is what we now do.

Let's begin by defining $\delta J_{\text {min }}$ for a single rotation, one can write $\sqrt{\frac{32}{27 \pi}}(M+\delta E)^{3 / 2}<J+\delta J$ and similarly for $\delta J_{\max }$. So the minimum threshold would be defined by

$\delta J_{\min }=\sqrt{\frac{8}{3 \pi}}\left(\frac{2}{3} M^{3 / 2} \epsilon^{2}+M^{1 / 2} \delta E+\frac{1}{4} M^{-1 / 2} \delta E^{2}\right)$.

Since $r_{+}=\left(\mu-b^{2}\right)^{1 / 2}$ and $b=\sqrt{\frac{8 M}{3 \pi}}\left(1-\epsilon^{2}\right)$ for a single rotation, $\delta J_{\max }$ yields

$\delta J_{\max }=\frac{r_{+}^{2}+b^{2}}{b} \delta E=\frac{\mu}{b} \delta E$

which, in turn, gives the upper bound up to $O\left(\epsilon^{2}\right)$,

$\delta J_{\max }=\sqrt{\frac{8}{3 \pi}}\left(1+\epsilon^{2}\right) M^{1 / 2} \delta E$.

So

$\Delta J=\sqrt{\frac{8}{3 \pi}}\left(M^{1 / 2} \epsilon^{2} \delta E-\frac{2}{3} M^{3 / 2} \epsilon^{2}-\frac{1}{4} M^{-1 / 2} \delta E^{2}\right)$,

which is clearly negative due to the dominant second and third terms. It is easy to see that the discriminant of the above quadratic is negative and hence $\Delta J<0$, signalling no overspinning of black hole. A five dimensional black hole with single rotation in contrast to its double rotation counterpart cannot be overspun by linear order accretion, and thereby it obeys CCC. As stated earlier, we have verified by following the non-linear analysis in Ref. [32] that as expected the result continues to hold good for non-linear regime as well.

\section{Conclusion}

We thus have that a five dimensional rotating black hole with only one rotation cannot be overspun to create a naked singularity even for a linear order test particle accretion while the opposite is true for the case of two rotations. For non-linear accretion, black hole can however never be overspun whether it has one or two rotations. Further it is well known that for a black hole with one rotation has no extremal limit in dimension $\geq 6$, and hence it could have arbitrary value of rotation parameter without risking overspinning and destruction of horizon.

Thus we can make a general pronouncement that a higher dimensional rotating black hole with dimension $>4$ with only single rotation always obeys the $\mathrm{CCC}$ in the weak form as it could not be overspun to destroy the horizon.

Let us note a black hole has one rotation in four dimension while it can have two rotations in five dimension. For linear accretion it appears that overspinning is only possible when black hole has maximum number of possible rotations; i.e. one for four dimension and two for five dimension. That is, a four dimensional rotating black hole and five dimensional black hole with two rotations could be overspun to destroy the horizon, thereby violating CCC in the weak form. On the other hand five dimensional black hole with single rotation can never be overspun. And black hole with single rotation in dimension greater than five cannot anyway be overspun as there exists no extremal limit.

The main result of this work could be couched as: All higher dimensional $(>4)$ rotating black holes with only one single rotation can never be overspun under test particle linear accretion and hence would always obey CCC in the weak form.

Acknowledgements BA acknowledges the Faculty of Philosophy and Science, Silesian University in Opava, Czech Republic, Inter-University Centre for Astronomy and Astrophysics, Pune, India, and Goethe University, Frankfurt am Main, Germany, for warm hospitality. This research is supported in part by Projects No. VA-FA-F-2-008 and No. MRB-AN-2019-29 of the Uzbekistan Ministry for Innovation Development, by the Abdus Salam International Centre for Theoretical Physics through Grant No. OEA-NT-01 and by an Erasmus + Exchange Grant between Silesian University in Opava and National University of Uzbekistan. 
Data Availability Statement This manuscript has no associated data or the data will not be deposited. [Authors' comment: This research is purely theoretical and has fundamental character. Thus, there is no data analysis.]

Open Access This article is distributed under the terms of the Creative Commons Attribution 4.0 International License (http://creativecomm ons.org/licenses/by/4.0/), which permits unrestricted use, distribution, and reproduction in any medium, provided you give appropriate credit to the original author(s) and the source, provide a link to the Creative Commons license, and indicate if changes were made. Funded by SCOAP ${ }^{3}$.

\section{References}

1. S.W. Hawking, R. Penrose, Proc. R. Soc. Lond. A 314, 529 (1970)

2. R. Penrose, Nuovo Cimento Rivista Serie 1, 252 (1969)

3. P.S. Joshi, International Series of Monographs on Physics (Clarendon (Oxford University Press), Oxford, 1993)

4. P.S. Joshi, Pramana 55, 529 (2000). arXiv:gr-qc/0006101

5. P.S. Joshi, The Story of Collapsing Stars: Black Holes, Naked Singularities, and the Cosmic Play of Quantum Gravity: (Oxford University Press, Oxford, 2015)

6. Z. Stuchlík, J. Schee, Class. Quantum Gravity 29, 065002 (2012)

7. R.S.S. Vieira, J. Schee, W. Kluźniak, Z. Stuchlík, M. Abramowicz, Phys. Rev. D 90, 024035 (2014). arXiv:1311.5820 [gr-qc]

8. Z. Stuchlík, J. Schee, Class. Quantum Gravity 31, 195013 (2014). arXiv:1402.2891 [astro-ph.HE]

9. B. Giacomazzo, L. Rezzolla, N. Stergioulas, Phys. Rev. D. 84, 024022 (2011). arXiv:1105.0122 [gr-qc]

10. R. Wald, Ann. Phys. (N.Y.) 82, 548 (1974)

11. N. Dadhich, K. Narayan, Phys. Lett. A 231, 335 (1997)

12. V.E. Hubeny, Phys. Rev. D 59, 064013 (1999). arXiv:gr-qc/9808043

13. T. Jacobson, T.P. Sotiriou, Phys. Rev. Lett. 103, 141101 (2009). arXiv:0907.4146 [gr-qc]

14. A. Saa, R. Santarelli, Phys. Rev. D 84, 027501 (2011). arXiv:1105.3950 [gr-qc]
15. M. Bouhmadi-López, V. Cardoso, A. Nerozzi, J.V. Rocha, Phys. Rev. D 81, 084051 (2010). arXiv: 1003.4295 [gr-qc]

16. Z. Li, C. Bambi, Phys. Rev. D 87, 124022 (2013). arXiv: 1304.6592 [gr-qc]

17. J.V. Rocha, R. Santarelli, Phys. Rev. D 89, 064065 (2014). arXiv: 1402.4840 [gr-qc]

18. S. Shaymatov, M. Patil, B. Ahmedov, P.S. Joshi, Phys. Rev. D 91, 064025 (2015). arXiv: 1409.3018 [gr-qc]

19. Y. Song, M. Zhang, D.-C. Zou, C.-Y. Sun, R.-H. Yue, Commun. Theor. Phys. 69, 694 (2018). arXiv:1705.01676 [gr-qc]

20. K. Düztaş, Class. Quantum Gravity 35, 045008 (2018). arXiv: 1710.06610 [gr-qc]

21. A.K. Mishra, S. Sarkar, arXiv e-prints (2019). arXiv:1905.00394 [gr-qc]

22. E. Barausse, V. Cardoso, G. Khanna, Phys. Rev. Lett. 105, 261102 (2010). arXiv:1008.5159 [gr-qc]

23. J.V. Rocha, V. Cardoso, Phys. Rev. D 83, 104037 (2011). arXiv:1102.4352 [gr-qc]

24. S. Isoyama, N. Sago, T. Tanaka, Phys. Rev. D 84, 124024 (2011). arXiv:1108.6207 [gr-qc]

25. P. Zimmerman, I. Vega, E. Poisson, R. Haas, Phys. Rev. D 87, 041501 (2013). arXiv:1211.3889 [gr-qc]

26. M. Colleoni, L. Barack, Phys. Rev. D 91, 104024 (2015). arXiv: 1501.07330 [gr-qc]

27. M. Colleoni, L. Barack, A.G. Shah, M. van de Meent, Phys. Rev. D 92, 084044 (2015). arXiv:1508.04031 [gr-qc]

28. J. Sorce, R.M. Wald, Phys. Rev. D. 96, 104014 (2017). arXiv: 1707.05862 [gr-qc]

29. B. Gwak, J. High Energy Phys. 9, 81 (2018). arXiv:1807.10630 [gr-qc]

30. K.S. Revelar, I. Vega, Phys. Rev. D. 96, 064010 (2017), arXiv: 1706.07190 [gr-qc]

31. B. Ge, Y. Mo, S. Zhao, J. Zheng, Phys. Lett. B 783, 440 (2018). arXiv:1712.07342 [hep-th]

32. J. An, J. Shan, H. Zhang, S. Zhao, Phys. Rev. D. 97, 104007 (2018). arXiv: 1711.04310 [hep-th]

33. R.C. Myers, M.J. Perry, Ann. Phys. (N.Y.) 172, 304 (1986)

34. S.W. Hawking, C.J. Hunter, M.M. Taylor-Robinson, Phys. Rev. D 59, 064005 (1999). arXiv:hep-th/9811056 